\title{
Psychiatric morbidity after screening for breast cancer
}

\author{
CHRISTINE DEAN, ${ }^{1}$ M MAUREEN ROBERTS, ${ }^{2}$ KATE FRENCH, ${ }^{2}$ AND SUSAN \\ ROBINSON ${ }^{2}$ \\ From the University Department of Psychiatry, ${ }^{1}$ Royal Edinburgh Hospital, and Breast Screening Clinic and \\ Department of Clinical Surgery, ${ }^{2}$ Edinburgh University
}

SUMMARY One hundred and thirty two women with normal breast screening results were interviewed six months after their attendance at the Edinburgh Breast Screening Clinic. Eight percent of women said screening had made them more anxious about developing breast cancer. Thirty eight percent said they were more aware of the disease since screening but they regarded this as advantageous. Seventy percent of the women were still practising breast self-examination. There was no difference in the psychiatric morbidity of the screened sample when compared with a matched random sample community control group. Neither was there any difference in the General Health Questionnaire case rates before and after screening. Screening does not appear to increase the prevalence of psychiatric morbidity. Twenty nine percent of the interview sample were examining their breasts more than once a month- $21 \%$ once a week or more. However, these frequent self-examiners did not have a greater prevalence of psychiatric morbidity than their matched controls.

There is increasing evidence that screening for breast cancer may lead to a worthwhile reduction in mortality from the disease, ${ }^{12}$ and a major trial to determine the effect of screening is under way in this country. ${ }^{3}$ However, screening may also have disadvantages, which should not be underestimated. ${ }^{4}$ For example, it is expensive when carried out on a population basis; some women undergo unnecessary treatment; and repeated mammography involves a radiation hazard, however small. A further disadvantage could be that screening causes an increase in anxiety about breast cancer among women who regularly attend and an increase in psychiatric morbidity. To our knowledge, this possibility has not been investigated. The study reported here was undertaken to examine the psychiatric morbidity of women who were screened within a randomir d trial of screening for breast cancer in Edinburg... ${ }^{3}$

\section{Methods}

In Edinburgh, a breast screening trial is currently underway which involves all women in the city aged between 45 and 65 years; half of the women are randomly allocated (by general practice, not by individual) for invitation for screening, the other half acting as controls. General practitioners are allowed to exclude from invitation those with severe physical or psychiatric morbidity. Women allocated for screening are sent a letter offering an appointment at the Screening Clinic. Overall, $67 \%$ of women have accepted the invitation and have undergone clinical and mammographic examination. Those who attend are also taught breast self-examination.

The current study of psychiatric morbidity comprises three groups of women: a 1 in 8 "interview sample" randomly selected from the women who were attending the breast screening clinic for the first time over a two month period (16 March 1981 to 14 May 1981); a "postal sample", comprising all the women attending for screening for the first time in the week 25 May to 1 June 1981; a "control sample" selected from a random sample of women in Edinburgh to match the "interview sample".

A total of 151 women were included in the "interview sample" and were asked to fill in a 30 -item general health questionnaire (GHQ) while waiting to be screened. The GHQ is a self rated screening questionnaire, which is used to screen for psychiatric morbidity in a population. The devisers of the instrument recommend the validation of the instrument against a psychiatric interview. For this reason we included an interview sample to enable us to decide the best cut off score on the questionnaire which would indicate the presence of probable psychiatric illness. The occupation of the woman and that of her husband were ascertained at this stage and 
also her willingness to be interviewed at home six months later. These women were then interviewed six months after screening by one of the two interviewers (KF and SR). The interview was semistructured and enquired about the woman's experience of screening and her subsequent attitude to breast cancer and breast self-examination. In addition, a semistructured psychiatric interview was carried out to assess the woman's current mental state. The instrument used was the psychiatric assessment schedule (PAS), ${ }^{6}$ which is a modification of the 40 -item present state examination (PSE). ${ }^{7}$ It allows the collection of symptom data in a reliable manner so that a research diagnostic criteria (RDC) ${ }^{8}$ diagnosis can be made. As well as being interviewed at six months after screening each woman also completed a 30 -item GHQ at that time.

The two interviewers were given a two week training in the use of the instrument (by CD). The training given to the interviewers was the same as that given to the interviewers in the Edinburgh Community Study ${ }^{9}$ which provided the control group. This included the rating of videotaped interviews, hospital patients, and patients in the community, all in a seminar setting. As in the community study, the interviewers completed PAS ratings of the two video tapes made by $C D$ at the end of their training. The interviewer ratings were then checked against the consensus ratings agreed between the tutors in the community study. All interviews during the study were audiotaped. Throughout the two months of the study CD listened to the interview tapes of those who were RDC cases and discussed the ratings with the interviewers.

The postal sample comprised all the women who attended the clinic for the first time in one week $(25$ May to 1 June 1981). Each woman completed a 30-item GHQ while waiting to be screened. The occupation of the woman and that of her husband were noted and she was asked about her willingness to complete a postal GHQ six months later. Altogether 158 women were invited to participate in this aspect of the study.

The interview sample from the screening clinic was compared with a control group, the control sample, comprising women randomly selected from the population of Edinburgh for another study, the details of which are published elsewhere. ${ }^{9}$ This study demonstrated a relation between psychiatric illness (as defined by the RDC used in this study) and marital status, social class, and employment status, but not age. However, there was a tendency for women aged 35-54 to have higher rates of psychiatric illness than those aged 55-65. Each woman in the screening clinic interview sample, therefore, was matched for marital status, social class, employment status, and age (under 55 or 55 and over) with a woman from the general population sample. One hundred and seventeen of the 132 women were successfully matched; no match was found for 15 of the sample. Fortunately, no woman turned out to be her own control. The randomly selected control sample had also been interviewed using the PAS. Although other interviewers were involved, they had all been trained in the same way (as detailed above), and the same tutor (CD) was involved in training both groups of interviewers. Both sets of interviewers were assessed by the same passing out procedure.

\section{Results}

Six women from the interview sample were subsequently excluded because they were recalled to the clinic for further investigations. Of the 145 women eligible for interview, four refused at the clinic to be interviewed six months later (they were not approached subsequently) and five refused an interview when approached. An interview was successfully obtained in 132 women (91\%).

Of the 132 women in the interview sample, 55\% were aged $45-54$ years, $89 \%$ were currently married, $60 \%$ were employed, $52 \%$ were middle class by the Goldthorpe and $\mathrm{Hope}^{10}$ criteria, and $70 \%$ were in Registrar General social classes I, II, and IIINM.

The women were classified according to their own occupation if they had a job or their father's (if they were unemployed, single, and living with him) or their husband's (if they were unemployed and living with him). Middle class is represented by Goldthorpe and Hope occupational groups 1 to 22 and working class by groups 23 to 26 .

Although our interview sample is a random sample of clinic attenders, clearly it is not a random sample of the population because not all invited women take up the offer of screening. During the period of the study, 1879 women between the ages of 45 and 60 were invited, of whom 1227 attended. Most of the women came from two general practices with a total eligible population of 1680 . One woman from the two practices concerned was excluded by her general practitioner because of psychiatric illness $(0.06 \%)$. Thirty three percent of women who were eligible for screening during the study period did not attend. It could be that the attenders have different psychiatric morbidity from the non-attenders so that the screened sample could be a biased sample from the morbidity point of view. This possibility was examined by checking the lists of women who were attenders and non-attenders during the study against the names on the Edinburgh Psychiatric Case Register (EPCR). This register records all psychiatric contacts, inpatient and outpatient. The name, 
address, and date of birth were used for matching purposes. Forty two of the 1227 attenders appeared on the EPCR $(3.4 \%)$ and 32 of the 652 non-attenders $(4.9 \%)$. The tendency for women who attend the clinic to have less psychiatric morbidity was not significant $\left(\chi^{2}=2.08 \mathrm{~ns}\right)$. We believe we have overcome this tendency by matching the screening sample with women from the random community sample, in terms of variables known to influence psychiatric morbidity-social class, marital status, employment status, and age.

\section{MOR B I DITY}

\section{Interview data}

Eighteen (15.4\%) of the interview sample who had a match (n=117) were RDC “cases" six months after attending the breast screening clinic compared with $20(17 \cdot 19 \%)$ of the matched control sample. As can be seen from the table, the type of cases in the two samples were almost identical. Twenty four $(20 \cdot 5 \%)$ of the interview sample were rated as having the PSE symptom of anxiety compared with $18(15.4 \%)$ of the control group.

Estimated prevalence of psychiatric disorder among the screening sample compared with a matched community sample

\begin{tabular}{lcl}
\hline$R D C$ diagnosis & $\begin{array}{l}\text { Screening sample } \\
(n=117)\end{array}$ & $\begin{array}{l}\text { Community sample } \\
(n=117)\end{array}$ \\
\hline Major depressive disorder & 7 & 9 \\
Minor depressive disorder & 6 & 6 \\
Generalised anxiety disorder & 4 & 5 \\
Panic disorder & 1 & 0 \\
Totals & 18 & 20 \\
\hline
\end{tabular}

\section{GHQ data}

As there were GHQ and interview data for women interviewed six months after screening, it was possible to estimate what cut-off score would best coincide with RDC "caseness". This method of obtaining the best cut-off is recommended by Goldberg in his manual. ${ }^{11}$ A cut-off of 3 on the GHQ was found to be the best, giving a sensitivity of $86 \%$ and a specificity of $91 \%$ (119 of the 132) $(90 \%$ were correctly labelled; there were 10 false positives and 3 false negatives). Therefore, when examining the GHQ data resulting from the postal part of the survey, a score of 4 or more was regarded as indicating a probable "case".

One hundred and thirty of the interview sample had GHQ data for before and after screening. One patient had not completed her questionnaire at the clinic and one was incomplete. Of the 158 in the postal sample, data were complete for $139(88 \%) ; 4$ were incomplete and 15 did not return their questionnaire.

We compared the GHQ "case rate" before and six months after screening. Twenty eight of the interview sample were GHQ cases before screening compared with 28 after screening. Eighteen of the postal sample were GHQ cases before screening compared with 19 after screening. These differences were not significant. The mean symptom score of the interview and postal sample together before screening was 1.766 compared with 2.048 after screening. The difference in scores before and after screening was not significant (Wilcoxon matched-pairs signed-rank test). Twenty women were regarded as "cases" before screening and were "non-cases" six months later. Twenty one women who were "non-cases" before screening were "cases" six months later. Twenty six women were "cases" on both occasions. The rest were "non-cases" on both occasions.

\section{REPORTED EXPERIENCE OF SCREENING}

Altogether $30 \%$ of women said they were made anxious by receiving the first letter of invitation, and $20 \%$ said they found the actual screening procedure to be anxiety provoking. Twice as many found the $x$-ray procedure upset them compared with the clinical examination. The screening procedure was found to be reassuring by $86 \%$ of women, and only 12 (9\%) complained of not feeling reassured by the time they left the clinic. Four of these 12 (33\%) women were cases at 12 months compared with 1 of the 12 matched controls. This difference was not significant. Six of these women had the PSE symptom of anxiety recorded compared with two of the control group, and this difference was significant $(p<0.05)$. The pre-screening GHQ scores of these women were high (a mean score of 4.75 ) and this was significantly higher than the rest of the interview sample (mean 1.95; $\mathrm{p}<0.05$; Mann Whitney $U$ test). Their mean score six months after screening was lower $(4 \cdot 17)$ than before screening but still higher than that of the rest of the interview sample.

Before screening, $40 \%$ of women said they sometimes worried about the possibility of breast cancer. Six months after screening $39 \%$ of women said they sometimes worried. Only 10 women (7.6\%) thought screening had made them more anxious about developing breast cancer (4 of these 10 were RDC cases six months after screening compared with none in the matched control group; this difference was significant FE $p<0.05)$. Six of these women had the PSE symptom of anxiety compared with none of the controls (FE $p<0.01)$. The prescreening GHQ scores (mean 2.3) of these women was not any higher than for the rest of the interview sample; nor was their mean GHQ score six months after screening 
(mean 3.0) higher than for the rest of the interview sample. The increase in mean GHQ score between the two times was not significant. This group of women differed from the rest of the interview sample in being more likely to be of lower social class $(60 \%$ were IIIM, IV or V compared with $37 \%$ of the rest, $\mathrm{p}<0 \cdot 1)$. They also had a tendency to be older, more were divorced, separated or widowed, and were more likely to be employed (90\%) than the rest of the interview sample.

More awareness of the disease as a result of screening was reported by $38 \%$ of women, but $93 \%$ of these thought that the increased awareness was a good thing.

\section{BREAST SELF-EXAMINATION}

Of the 132 women interviewed, $88(67 \%)$ said they were still practising self-examination six months later. Only $31(23.5 \%)$ were carrying it out at monthly intervals, $38(29 \%)$ performing it more often, $27(20.5 \%)$ of them once or more weekly. However, only two women said breast self-examination made them anxious (and neither of these was an RDC case six months after screening), the majority (90\%) claiming that they were reassured by the procedure. Women who examined their breast more frequently than once a month did not have a higher case rate than the matched control group; there were seven cases $(18 \%)$ in both groups. There was no difference either in the number of women who had the PSE symptom of anxiety: nine in the screened sample compared with eight in the control group. They did have a non-significant tendency to be of lower social class than the rest of the interview sample, and more of them were divorced, widowed or separated.

\section{Discussion}

Most women did not find that going to the breast screening clinic made them feel anxious about breast cancer. The numbers worrying about breast cancer before and after screening were about the same. However, this assessment was done retrospectively. In a random sample of the population ${ }^{12}$ only $5 \%$ of women spontaneously mentioned breast cancer as an illness they personally worried about compared with $40 \%$ of women in this study before and six months after screening. However, the screened sample were asked directly if they worried about breast cancer, and this could account for the difference or it could be that screening had raised awareness about breast cancer.

Screening does not appear to increase psychiatric morbidity. There was no difference in the number of psychiatric cases established at interview six months after screening when compared with a matched sample of the population. It is possible that because of a type II error a small real difference between the screened population and the control group was not demonstrated. As already mentioned above, there was a non-significant tendency for those who came for screening to have a lower psychiatric morbidity than those who did not. Other studies have shown that non-attenders are of a lower social class than attenders, ${ }^{13} 14$ and those of a lower social class do have a higher psychiatric morbidity. ${ }^{9}$ However, we believe we have controlled for this bias by matching for social class, marital status, age, and employment status. Using the GHQ postal data gave us a larger sample size $(n=269)$. However, even with a sample size of 269 a type II error may have prevented us from demonstrating a small but real difference in psychiatric morbidity before and after screening. Although the GHQ refers to symptoms in the few weeks before completing the questionnaire, the GHQ was, in this study, completed at a stressful time, that is, immediately before a first breast screening. This may have inflated the prescreening scores and may have concealed an increased symptom score after screening compared with the patients' true prescreening state.

Women in whom an abnormality was found and who had to return to the clinic were not included in the study. Thirty eight per 1000 women were asked to return $(6 / 1000$ women prove to have cancer: $12 / 1000$ have biopsies for benign lesions: the rest? have other investigations). It is not possible from our study to comment on the psychiatric morbidity after screening in this group; it requires a further study.

A small group of women (9\%) complained of not being reassured by the time they left the clinic. They did not have a higher case rate than controls at six months but did have a higher number declaring the PSE symptom of anxiety. However, these women had higher GHQ scores before screening, and the likely explanation of their not feeling reassured is that they were particularly anxious women.

We found that large numbers of women were carrying out breast self-examination more frequently than recommended (more than once a month). However, these women did not have an increased psychiatric case rate nor did they have increased rates of anxiety. It could be that they had failed to understand the instructions given to them at the screening clinic.

A small percentage of women (8\%) felt that breast screening had made them feel anxious about developing breast cancer. This small group of women did in fact have a higher psychiatric morbidity than their matched control group. It could be that a small subgroup of women are vulnerable and that 
screening has a detrimental effect on them from the psychiatric point of view. However, taking the sample as a whole, our data do not support the suggestion that screening for breast cancer increases psychiatric morbidity.

We acknowledge the work of Drs N B Kreitman, P G Surtees, J G Ingham, P Mc C Miller, and S P Sashidharan of the MRC Unit for Epidemiological Studies in Psychiatry, Edinburgh, who were involved with $C D$ in the community study which provided us with a control group for the screening sample. The Edinburgh Screening Project is funded by the Scottish Home and Health Department and the Cancer Research Campaign.

Correspondence and reprints to: $\mathrm{Dr} \mathrm{C}$ Dean, Consultant Psychiatrist, Manchester Royal Infirmary.

\section{References}

${ }^{1}$ Shapiro S, Venet W, Strax P, Venet L, Roeser R. Ten to fourteen year effect of screening on breast cancer mortality. J Natl Cancer Inst 1982; 69: 349-55.

${ }^{2}$ Tabar L, Fagerber CJG, Gad A, et al. Reduction in mortality from breast cancer after mass screening with mammography. Lancet 1985; i: 829-32.
${ }^{3}$ UK Breast Cancer Detection Working Group. Trial of early detection of breast cancer; description of method. Br J Cancer 1981; 44: 618-27.

${ }^{4}$ Smith A. Preserving the future-the potential of population screening. Health and Hygiene 1977; 1: 147-51.

${ }^{5}$ Roberts MM, Alexander FE, Anderson TJ, Forrest APM, Hepburn W, Kirkpatrick AE. A randomised trial of screening for breast cancer. Br J Cancer 1984; 50: 1-6.

${ }^{6}$ Dean C, Surtees PG, Sashidharan SP. Comparison of research diagnostic systems in an Edinburgh community sample. Br J Psychiatry 1983; 142: 247-56.

${ }^{7}$ Wing JK, Cooper JE, Sartorius N. The measurement and classification of psychiatric symptoms. Cambridge: Cambridge University Press, 1974.

${ }^{8}$ Spitzer RL, Endicott J, Robins E. Research diagnostic criteria; rationale and reliability. Arch Gen Psychiatry 1978; 35: 773-82.

${ }^{9}$ Surtees PG, Dean C, Ingham JG, Kreitman NB, Miller PMcC, Sashidharan SP. Psychiatric disorder in women from an Edinburgh community; associations with demographic factors. $\mathrm{Br} J$ Psychiatry 1983; 142: 238-46.

${ }^{10}$ Goldthorpe J, Hope K. The social grading of occupations: a new approach scale. London: Oxford University Press, 1974.

${ }^{11}$ Goldberg D. Manual of the General Health Questionnaire. Horsham: NFER Publishing Co, 1978.

${ }^{12}$ Roberts MM, French K, Duffy J. Breast cancer and breast self-examination; what do Scottish women know? Soc Sci Med 1984; 18: 791-7.

${ }^{13}$ French K, Porter AMD, Robinson SE, McCallum F, Howie JGR, Roberts MM. Attendance at a breast screening clinic; a problem of administration or attitudes. $\mathrm{Br} \mathrm{Med} J$ 1982; 284: 617-20.

${ }^{14}$ MacLean U, Sinfield D. Women who decline screening; report to Health Services Research Committee. J Epidemiol and Comm Med 1984; 38: 278-83. 